\title{
Einstein as armchair detective: The case of stimulated radiation
}

\author{
Vasant Natarajan* \\ Department of Physics, Indian Institute of Science, Bangalore 560 012, India
}

July 22, 2013

\begin{abstract}
Einstein was in many ways like a detective on a mystery trail, though in his case he was on the trail of nature's mysteries and not some murder mystery! And like all good detectives he had a style. It consisted of taking facts that he knew were correct and forcing nature into a situation that would contradict this established truth. In this process she would be forced to reveal some new truths. Einstein's 1917 paper on the quantum theory of radiation is a classic example of this style and enabled him to predict the existence of stimulated radiation starting from an analysis of thermodynamic equilibrium between matter and radiation.
\end{abstract}

Keywords: Radiation, emitter-absorber interaction, radiation reaction.

Einstein is rightly regarded as one of the greatest scientific geniuses of all time. Perhaps the most amazing and awe-inspiring feature of his work was that he was an "armchair" scientist, not a scientist who spent long hours in a darkened laboratory conducting delicate experiments, but one who performed gedanken (thought) experiments while sitting in his favourite chair that nevertheless advanced our understanding of nature by leaps and bounds. Two of his greatest contributions are the special theory of relativity and the general theory of relativity, both abstract creations of his remarkable intellect. They stand out as scientific revolutions that completely changed our perceptions of nature - of space and time in the case of the special theory and of gravity in the case of the general theory. It might be argued that the special theory of relativity was necessitated by experimental facts such as the constancy of the speed of light, but the general theory was almost completely a product of Einstein's imagination. For a person to have achieved one revolution in his lifetime is great enough, but two revolutions seems quite supernatural.

But is it really so magical? While it is certain that Einstein was a oneof-a-kind genius, is it at least possible to understand the way in which his

*email: vasant@physics.iisc.ernet.in 
mind tackled these problems? I think the answer is yes, because deep inside Einstein was like a detective hot on a mystery trail, of course not one solving murder mysteries but one trying to unravel the mysteries of nature. Any keen follower of murder mysteries knows that there are two types of detectives: those who get down on their hands and knees looking for some microscopic piece of clinching evidence at the scene of the crime, and the second type of "armchair detectives" who seem to arrive at the solution just by thinking logically about the possibilities. Einstein was most certainly of the second kind, and true to this breed, he had his own modus operandi. In simple terms, his technique was to imagine nature in a situation where she contradicted established truths, and revealed new truths in the process. As a case in point, we will look at Einstein's 1917 paper titled "On the quantum theory of radiation" where he predicted the existence of stimulated emission. While Einstein will always be remembered for his revolutionary relativity theories, his contributions to the early quantum theory are certainly of the highest calibre and the 1917 paper is a classic.

It is useful to first set the paper in its historical perspective. By the time Einstein wrote this paper, he had already finished most of his work on the relativity theories. He had earlier done his doctoral thesis on Brownian motion and was a pioneer of what is now called statistical mechanics. He was thus a master at using thermodynamic arguments. He was one of the earliest scientists to accept Planck's radiation law and its light quantum hypothesis. He had already used it in 1905 for his explanation of the photoelectric effect. He was also aware of Bohr's theory of atomic spectra and Bohr's model of the atom, which gave some explanation for why atoms emitted radiation in discrete quanta. What he did not know in 1917 was any of the formalism of quantum mechanics, no Schrödinger equation and not the de Broglie hypothesis for wave nature of particles that we learn in high school these days. Despite this, Einstein was successful in predicting many new things in this paper.

Let us now see what Einstein's strategy in this paper is. He is attempting to understand the interaction between atoms and radiation from a quantum mechanical perspective. For this, he imagines a situation where a gas of atoms is in thermal equilibrium with radiation at a temperature $T$. The temperature $T$ determines both the Maxwell-Boltzmann velocity distribution of atoms and the radiation density $\rho$ at different frequencies through Planck's law. He assumes that there are two quantum states of the atom $Z_{n}$ and $Z_{m}$, whose energies are $\varepsilon_{n}$ and $\varepsilon_{m}$ respectively, and which satisfy the inequality $\varepsilon_{m}>\varepsilon_{n}$. The relative occupancy $W$ of these states at a temperature $T$ depends on the Boltzmann factor as follows:

$$
\begin{aligned}
W_{n} & =p_{n} \exp \left(-\varepsilon_{n} / k t\right) \\
W_{m} & =p_{m} \exp \left(-\varepsilon_{m} / k t\right)
\end{aligned}
$$

where $p_{n}$ is a number, independent of $T$ and characteristic of the atom and its $n$th quantum state, called the degeneracy or "weight" of the particular state. Similarly, $p_{m}$ is the weight of the $m$ th state.

Einstein then makes the following basic hypotheses about the laws governing 
the absorption and emission of radiation:

1. Atoms in the upper $(m)$ state make a transition to the lower $(n)$ state by spontaneous emission. The probability $d W$ that such a transition occurs in the time $d t$ is given by:

$$
d W=A_{m}^{n} d t
$$

$A_{n}^{m}$ in modern terminology is called the Einstein $A$ coefficient. Since this process is intrinsic to the system and is not driven by the radiation field, it has no dependence on the radiation density.

2. Atoms in the lower state make a transition to the upper state by absorbing radiation. The probability that such a transition occurs in the time $d t$ is given by:

$$
d W=B_{n}^{m} \rho d t
$$

$B_{m}^{n}$ is now called the Einstein $B$ coefficient. The absorption process is driven by the radiation field, therefore the probability is directly proportional to the radiation density $\rho$ at frequency $\nu$.

3. The two postulates above seem quite reasonable. Now comes his new postulate, that there is a third process of radiative transition from the upper state to the lower state, namely stimulated emission, driven by the radiation field. By analogy with the probability for absorption, the probability for stimulated emission is:

$$
d W=B_{m}^{n} \rho d t
$$

Einstein calls the processes in both 2 and 3 as "changes of state due to irradiation". We will see below how he is forced to include postulate 3 in order to maintain thermodynamic equilibrium.

The main requirement of thermodynamic equilibrium is that the occupancy of atomic levels given by Eqs. 1 and 2 should not be disturbed by the absorption and emission processes postulated above. Therefore the number of absorption processes (type 2) per unit time from state $n$ into state $m$ should equal the number of emission processes (type 1 and 3 combined) out of state $m$ into state $n$. This is called detailed balance. Since the number of processes from a given state occurring in a time $d t$ is given by the occupancy of that state times the probability of a transition, the detailed balance condition is written as:

$$
p_{n} \exp \left(-\varepsilon_{n} / k T\right) B_{n}^{m} \rho=p_{m} \exp \left(-\varepsilon_{m} / k T\right)\left(B_{m}^{n} \rho+A_{m}^{n}\right)
$$

Notice the importance of the third hypothesis about stimulated emission to make the equation consistent. If one does not put that in, the equation becomes:

$$
p_{n} \exp \left(-\varepsilon_{n} / k T\right) B_{n}^{m} \rho=p_{m} \exp \left(-\varepsilon_{m} / k T\right) A_{m}^{n}
$$

which clearly will not work. At high temperatures, when the Boltzmann factor makes the occupancy of the two levels almost equal, the rate of absorption on 
the LHS increases with temperature as the radiation density increases. But the rate of emission on the RHS does not increase because spontaneous emission is independent of the radiation density. Thermodynamic equilibrium will therefore not be maintained. This is vintage Einstein: he imagines a situation that forces a contradiction with what he "knows", namely thermal equilibrium, and uses it to obtain a new result, namely stimulated emission during radiative transfer.

With the grace and confidence of an Olympic hurdler, Einstein now moves on to make quantitative predictions based on the bold new hypothesis. First he uses the high temperature limit to derive a relation between the coefficients for absorption and stimulated emission. Under the reasonable assumption that $\rho \rightarrow \infty$ as $T \rightarrow \infty$, the spontaneous emission term on the RHS of Eq. 6] can be neglected at high temperatures. From this, it follows that:

$$
p_{n} B_{n}^{m}=p_{m} B_{m}^{n}
$$

By substituting this result in Eq. 6. Einstein obtains a new, simple derivation of Planck's law:

$$
\rho=\frac{A_{m}^{n} / B_{m}^{n}}{\exp \left[\left(\varepsilon_{m}-\varepsilon_{n}\right) / k T\right]-1}
$$

Notice that he will not get the correct form of this law if he did not have the stimulated emission term in Eq. 6. Another reason for him to be confident that his three hypotheses about absorption and emission are correct. He then compares the above expression for $\rho$ with Wien's displacement law:

$$
\rho=\nu^{3} f(\nu / T)
$$

to obtain

$$
\frac{A_{m}^{n}}{B_{m}^{n}}=\alpha \nu^{3}
$$

and

$$
\varepsilon_{m}-\varepsilon_{n}=h \nu
$$

with constants $\alpha$ and $h$. The second result is well known from the Bohr theory of atomic spectra. Einstein is now completely sure that his three hypotheses about radiation transfer are correct since he has been able to derive both Planck's law and Bohr's principle based on these hypotheses.

Einstein does not stop here. He now considers how interaction with radiation affects the atomic motion in order to see if he can predict new features of the momentum transferred by radiation. Earlier he had argued that thermal equilibrium demands that the occupancy of the states remain undisturbed by interaction with radiation. Now he argues that the Maxwell-Boltzmann velocity distribution of the atoms should not be disturbed by the interaction. In other words, the momentum transfer during absorption and emission should result in the same statistical distribution of velocities as obtained from collisions. From kinetic theory, we know that the Maxwell velocity distribution results in an average kinetic energy along each direction given by:

$$
\frac{1}{2} M\left\langle v^{2}\right\rangle=\frac{1}{2} k T
$$


This result should remain unchanged by the interaction with radiation.

To calculate the momentum change during radiative transfer, Einstein brings into play his tremendous insight into Brownian motion. As is now well known from the Langevin equation, he argues that the momentum of the atom undergoes two types of changes during a short time interval $\tau$. One is a frictional or damping force arising from the radiation pressure that systematically opposes the motion. The second is a fluctuating term arising from the random nature of the absorption-emission process. It is well known from Brownian motion theory that the atoms would come to rest from the damping force if the fluctuating term were not present. Thus, if the initial momentum of the atom is $M v$, then after a time $\tau$, the momentum will have the value:

$$
M v-R v \tau+\Delta
$$

where the second term is the damping term and the last term is the fluctuating term. If the velocity distribution of the atoms at temperature $T$ is to remain unchanged by this momentum transfer process, the average of the above quantity (Eq. 12) must be equal to $M v$, and the mean values of the squares of these quantities must also be equal:

$$
\left\langle(M v-R v \tau+\Delta)^{2}\right\rangle=\left\langle(M v)^{2}\right\rangle
$$

Since we are only interested in the systematic effect of $v$ on the momentum change due to interaction with radiation, $v$ and $\Delta$ can be regarded as independent statistical processes and the average of the cross term $v \Delta$ can be neglected. This yields:

$$
\left\langle\Delta^{2}\right\rangle=2 R M\left\langle v^{2}\right\rangle \tau
$$

To maintain consistency with kinetic theory, the value of $\left\langle v^{2}\right\rangle$ in the above equation must be the same as the one in Eq. 13. Thus:

$$
\frac{\left\langle\Delta^{2}\right\rangle}{\tau}=2 R k T
$$

This is the equation that will tell Einstein if his hypotheses about momentum transfer are correct. In other words, he assumes that the radiation density is given by Planck's law, and calculates $R$ and $\left\langle\Delta^{2}\right\rangle$ based on some hypotheses about momentum transfer during radiative processes. If the hypotheses are valid, the above equation should be satisfied identically in order not to contradict thermal equilibrium.

His main hypothesis about momentum transfer is that, if the photon behaves like a localized packet of energy $E$, it must also carry directional momentum of $E / c$. Without going into the details, I just outline the approach he uses for calculating $R$ and $\left\langle\Delta^{2}\right\rangle$. For calculating $R$, he uses the following argument. In the laboratory frame in which the atom has a velocity $v$, the radiation is isotropic. But in the rest frame of the atom, the radiation is anisotropic because of the Doppler shift. This gives rise to a velocity dependent radiation density and a velocity dependent probability of absorption and stimulated emission (from 
Eqs. (4) and (5). The average momentum transferred to the atom is calculated from the modified rates of absorption stimulated emission, thus yielding $R$. $R$ does not depend on the rate of spontaneous emission because spontaneous emission occurs independently of the radiation field and is therefore isotropic in the rest frame of the atom. Calculating $\left\langle\Delta^{2}\right\rangle$ is relatively simpler. If each absorption or emission process gives a momentum kick of $E / c$ in a random direction, the mean square momentum after $\ell$ kicks is simply $\ell \times(E / c)^{2} . \ell$ is equal to twice the number of absorption processes taking place in the time $\tau$ since each absorption process is followed by an emission process. Using this approach, Einstein calculates $R$ and $\left\langle\Delta^{2}\right\rangle$. He shows that Eq. 17 is satisfied identically when these values are substituted, which implies that the velocity distribution from kinetic theory is not disturbed if and only if momentum exchange with radiation occurs in units of $E / c$ in a definite direction.

He thus concludes the paper with the following observations. There must be three processes for radiative transfer, namely absorption, spontaneous emission, and stimulated emission. Each of these interactions is quantized and takes place by interaction with a single radiation bundle. The radiation bundle (which we today call a photon) carries not only energy of $h \nu$ but also momentum of $h \nu / c$ in a well defined direction. The momentum transferred to the atom is in the direction of propagation for absorption and in the opposite direction for emission. And finally, ever loyal to his dislike for randomness in physical laws ("God does not play dice!"), he concludes that one weakness of the theory is that it leaves the duration and direction of the spontaneous emission process to "chance". However, he is quick to point out that the results obtained are still reliable and the randomness is only a defect of the "present state of the theory".

What far reaching conclusions starting from an analysis of simple thermodynamic equilibrium. This is a truly great paper in which we see two totally new predictions. First, he predicts the existence of stimulated emission. And to top that, for the first time since Planck introduced radiation quanta, he shows that each quantum carries well defined momentum. He shows that the directional momentum is present even in the case of spontaneous emission. Thus an atom cannot decay by emitting "outgoing radiation in the form of spherical waves" with no momentum recoil.

Today his conclusions about momentum transfer during absorption and emission of radiation have been abundantly verified. Equally well verified is his prediction of stimulated emission of radiation. Stimulated emission is the mechanism responsible for operation of the laser, which is used in everything from home computers and CD players to long distance communication systems. Stimulated emission, or more correctly stimulated scattering, underlies our understanding of the phenomenon of Bose-Einstein condensation. It plays an important role in the explanation of superconductivity and superfluidity. The two predictions, momentum transfer from photons and stimulated emission, are particularly close to my heart because they play a fundamental role in one of my areas of research, namely laser cooling of atoms. In laser cooling, momentum transfer from laser photons is used to cool atoms to very low temperatures of a few millionths of a degree above absolute zero. Perhaps fittingly, it is the ran- 
domness or "chance" associated with the spontaneous emission process which he disliked so much that is responsible for the entropy loss associated with cooling. In other words, as the randomness from the atomic motion gets reduced by cooling, it gets added to the randomness in the radiation field through the spontaneous emission process, thus maintaining consistency with the second law of thermodynamics.

\section{Conclusions}

We have seen how Einstein was able to use the principle of thermodynamic equilibrium to imagine a situation where radiation and matter were in dynamical equilibrium and from that predict new features of the radiative transfer process. As mentioned before, this was a recurring theme in his work, a kind of modus operandi for the great "detective". In his later writings, he said that he always sought one fundamental governing principle from which he could derive results through these kind of arguments. He found such a principle for thermodynamics, namely the second law of thermodynamics, which states that it is impossible to build a perpetual motion machine. He showed that the second law was a necessary and sufficient condition for deriving all the results of thermodynamics. His quest in the last four decades of his life was to geometrize all forces of nature. In this quest, he felt that he had indeed found the one principle that would allow him to do this uniquely, and this was the principle of relativity:

the laws of physics must look the same to all observers no matter what their state of motion.

He had already used this principle to geometrize gravity in the general theory of relativity. His attempts at geometrizing electromagnetic forces remained an unfulfilled dream, but that is a story for another day. 


\section{Appendix. Examples of gedanken experiments}

We present two examples of gedanken experiments that illustrate the Einstein technique for arriving at new results. Both of these experiments yield results associated with the general theory of relativity, but are so simple and elegant that they can be understood without any knowledge of the complex mathematical apparatus needed for the general theory. The first experiment is due to Einstein himself, while the second is due to Hermann Bondi.

Example 1. This is a thought experiment devised by Einstein to arrive at the conclusion that the general theory of relativity is an extension of the special theory which requires curved spacetime, or spacetime in which the rules of plane (Euclidean) geometry do not apply. The "known" facts are the results of special theory of relativity applicable to inertial systems, and the equivalence principle which states that inertial mass is exactly equal to gravitational mass. Einstein's argument proceeds as follows.

Imagine two observers or coordinate systems $O$ and $O^{\prime}$. Let the $z^{\prime}$-axis of $O^{\prime}$ coincide with the $z$-axis of $O$, and let the system $O^{\prime}$ rotate about the $z$-axis of $O$ with a constant angular velocity (see Figure 1). Thus $O$ is an inertial system where the laws of special relativity apply, while $O^{\prime}$ is a non-inertial system. Imagine a circle drawn about the origin in the $x^{\prime} y^{\prime}$ plane of $O^{\prime}$ with some given diameter. Imagine, further, that we have a large number of rigid rods, all identical to each other. We lay these rods in series along the circumference and the diameter of the circle, at rest with respect to $O^{\prime}$. If the number of rods along the circumference is $U$ and the number of rods along the diameter is $D$, then, if $O^{\prime}$ does not rotate with respect to $O$, we have (from plane geometry)

$$
\frac{U}{D}=\pi
$$

However, if $O^{\prime}$ rotates, we get a different result. We know from special relativity that, relative to $O$, the rods on the circumference undergo Lorentz contraction while the rods along the diameter do not undergo this contraction (the relative motion is perpendicular to the diameter). Therefore, we are led to the unavoidable conclusion that

$$
\frac{U}{D}>\pi
$$

i.e. the laws of configuration of rigid bodies with respect to $O^{\prime}$ is not in accordance with plane geometry. If, further, we place two identical clocks, at rest with respect to $O^{\prime}$, one at the periphery and one at the centre of the circle, then with respect to $O$ the clock at the periphery will go slower than the clock at the centre (from special relativity, moving clocks go slower). A similar conclusion will be reached by $O^{\prime}$, i.e. the two clocks go at different rates.

We thus see that space and time cannot be defined with respect to $O^{\prime}$ as they were defined in special theory of relativity for inertial systems. But, according to the equivalence principle, $O^{\prime}$ can also be considered a system at rest with respect to which there is a gravitational field (corresponding to the centrifugal 


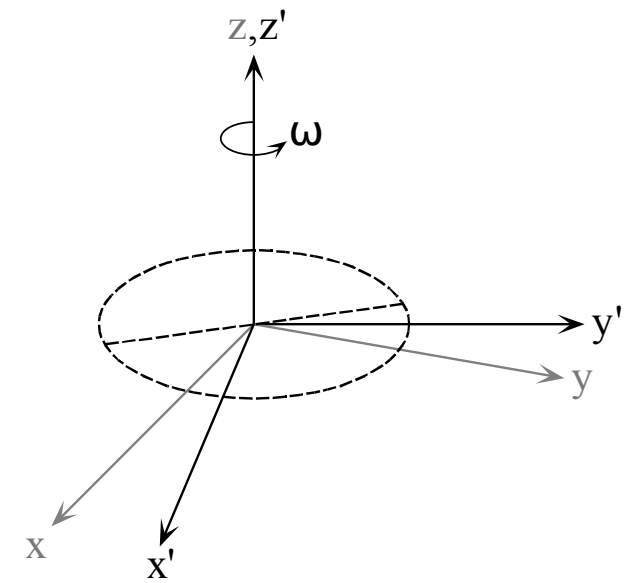

Figure 1: Coordinate systems with relative rotation between them.

force field and the Coriolis force field). We therefore arrive at the following remarkable result: the gravitational field influences and even determines the geometry of the space-time continuum, and this geometry is not Euclidean. From this conclusion, Einstein goes on to develop a curved spacetime theory of gravitation.

Example 2. This example illustrates the use of a thought experiment to calculate the difference in rates between two clocks placed at different gravitational potentials, called the gravitational redshift. We have already seen in the first example how the rate of the clock at the periphery differs from the rate of the clock at the centre. Here, we derive a quantitative value for this difference using an Einstein-like gedanken experiment, first conceived by Bondi. The "known" things are the second law of thermodynamics and the special relativistic energymass relationship, $E=m c^{2}$. The argument proceeds as follows.

Imagine a series of buckets on a frictionless pulley system, as shown in Figure 2. Each bucket contains an atom capable of absorbing or emitting a photon of energy $h \nu$. The system is in a uniform gravitational field with acceleration $g$. If the photon frequency were unaffected by the gravitational field, we can operate the system as a perpetual motion machine in the following way. Imagine that the pulleys rotate clockwise and that all the atoms on the left are in the ground state and the atoms on the right are in the excited state. The lifetime in the excited state is such that, on average, every time a bucket reaches the bottom the atom inside decays to the ground state and emits a photon. Suitable reflectors direct this photon to the corresponding bucket at the top so that the atom inside absorbs the photon and goes into the excited state. All the excited state atoms on the right have more energy and, from the relation $E=m c^{2}$, are therefore heavier by an amount $\Delta m=h \nu / c^{2}$. The heavier masses are accelerated down by the gravitational field and the system remains in perpetual motion. The 


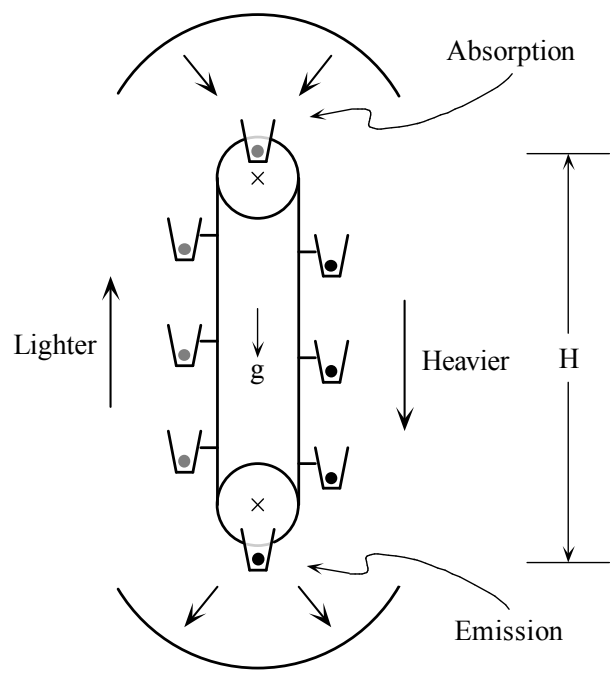

Figure 2: Bondi's perpetual motion machine. The buckets on the right side contain atoms that have higher energy and are thus heavier than the atoms on the left side. When a bucket reaches the bottom, the atom inside emits a photon which is absorbed by the corresponding atom in the top bucket. The heavier buckets on the right keep falling down in the gravitational field and their gravitational energy can be converted to useful work. The resolution to the paradox is that the photon absorbed at the top has a lower frequency than the photon emitted at the bottom.

excess gravitational potential energy can be converted to unlimited useful work, in violation of the second law of thermodynamics.

The solution to the paradox lies in postulating that the frequency of the photon emitted by the atom at the bottom is not the same as the frequency of the photon when it reaches the top. Let the two frequencies be $\nu$ and $\nu^{\prime}$ respectively. Then the additional mass for the atom at the top by absorbing a photon of frequency $\nu^{\prime}$ is $h \nu^{\prime} / c^{2}$, and the potential energy of this excess mass at a height $H$ between the two buckets is $h \nu^{\prime} / c^{2} \times g H$. To maintain consistency with the second law of thermodynamics, this excess energy should exactly compensate for the loss in energy of the photon as its frequency changes from $\nu$ to $\nu^{\prime}$ :

$$
\frac{h \nu^{\prime}}{c^{2}} g H=h\left(\nu-\nu^{\prime}\right)
$$

which yields

$$
\frac{\nu^{\prime}-\nu}{\nu^{\prime}}=-\frac{g H}{c^{2}}
$$

i.e. the relative frequency shift is given by $g H / c^{2}$ and is negative (redshift) at the location where the gravitational potential is higher. The shift can be understood from the fact that the photon is also affected by the gravitational 
field and therefore loses energy as it climbs up the potential. Since the photon always travels at the speed $c$, it loses energy by changing its frequency. This result explains why, in the first example, the clock at the centre goes slower than the clock in the periphery according to $O^{\prime}$. With respect to $O^{\prime}$, there is a gravitational field (corresponding to the centrifugal force) pointing away from the centre. The clock at the centre is at a higher gravitational potential and hence goes slower.

The gravitational redshift on the surface of the earth is very tiny at any reasonable height, but it was experimentally verified in a remarkable experiment by Pound and Rebka in 1959. They measured the frequency shift between the top and bottom of a building at Harvard University, a height difference of about $25 \mathrm{~m}$. The relative frequency shift measured was a tiny 3 parts in $10^{14}$, consistent with the above calculation!

\section{Author introduction:}

Vasant Natarajan is at the Department of Physics, Indian Institute of Science. His current research involved trapping of atoms to carry out high precision tests of fundamental physics. He has earlier worked on high precision mass spectrometry and on the focussing of atomic beams by laser fields. 